TRANSACTIONS OF THE

AMERICAN MATHEMATICAL SOCIETY

Volume 329, Number 1, January 1992

\title{
A RELATIONSHIP BETWEEN THE JONES AND KAUFFMAN POLYNOMIALS
}

\author{
CHRISTOPHER KING
}

\begin{abstract}
A simple relationship is presented between the Kauffman polynomial of a framed link $L$ and the Jones polynomial of a derived link $\widetilde{L}$. The link is $\widetilde{L}$ obtained by splitting each component of $L$ into two parallel strands, using the framing to determine the linking number of the strands. The relation is checked in several nontrivial examples, and a proof of the general result is given.
\end{abstract}

\section{INTRODUCTION}

Shortly after $\mathrm{V}$. Jones discovered a new polynomial invariant $V_{L}(t)$ for oriented links [1], several generalizations were found. One of these was the twovariable Kauffman polynomial $F_{L}(a, x)$ [2]. It was quickly realized that this polynomial, when restricted to a one-dimensional subset of its parameter space, reproduces the Jones polynomial [3]. Specifically,

$$
V_{L}(t)=F_{L}\left(t^{-3 / 4},-\left(t^{-1 / 4}+t^{1 / 4}\right)\right) .
$$

In this paper we introduce a new relationship between the Jones and Kauffman polynomials. It differs substantially from the relation just stated, because it relates the Jones and Kauffman polynomials of different links. It is easiest to state for a knot, so we will explain this case first.

Suppose $K$ is a knot, that is, an embedding of $S^{1}$ in $\mathbb{R}^{3}$. Imagine now that this knot is constructed from a closed piece of string, and that the string is itself composed of two parallel, braided strands. As long as the strands are stuck together, we perceive a single closed loop. However, if the strands become unstuck and then separate, we obtain a link $\widetilde{K}$ with two components. For example, Figure 1.1 shows the unknot and a link obtained from it by the procedure described. The main result in this paper is a relationship between the Kauffman polynomial of $K$ and the Jones polynomial of $\widetilde{K}$.

There is some ambiguity in the procedure described above. Firstly, we will be concerned throughout with oriented links, and so we must specify how the orientations of $K$ and $\widetilde{K}$ are related. We will always assume that the strands in $\widetilde{K}$ are oriented so that when they are stuck together to make the knot $K$, their directions are parallel and agree with the orientation of $K$. The link $\widetilde{U}$ in Figure 1.1 illustrates this.

Received by the editors November 9, 1989.

1980 Mathematics Subject Classification (1985 Revision). Primary 57M25; Secondary 81E13. 

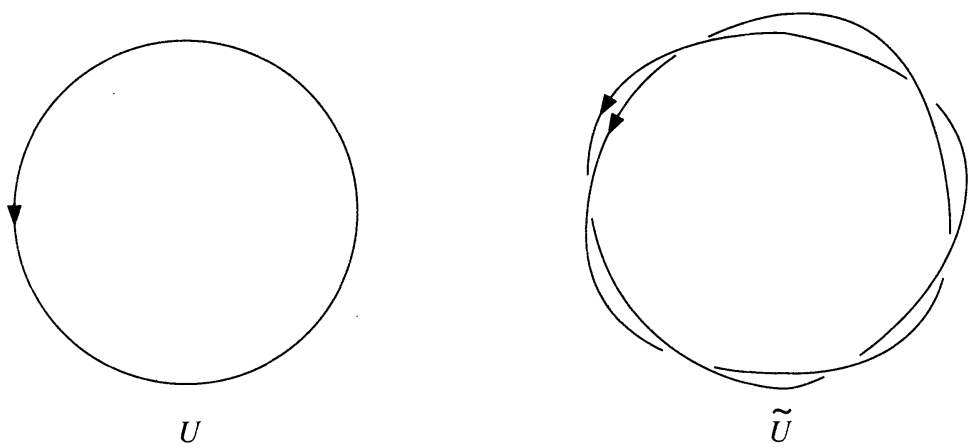

FIgURE 1.1. The unknot $U$ and a link $\widetilde{U}$

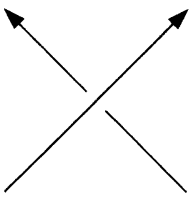

$\varepsilon=+1$

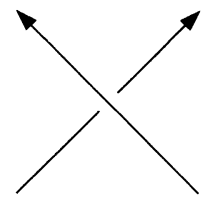

$\varepsilon=-1$

FIGURE 1.2. Conventions for the linking number

Secondly, we must specify how the two strands in $\widetilde{K}$ are braided together to make the single loop of string. This braiding is completely described by an integer $f$, which is the Gauss linking number of the two strands. Recall that the linking number of two oriented knots $K_{1}, K_{2}$ is defined as follows [2]. In a regular projection of the link, we assign a number $\varepsilon(p)= \pm 1$ to each point $p$ where $K_{1}$ and $K_{2}$ cross, according to the conventions in Figure 1.2. The linking number is then defined to be

$$
w\left(K_{1}, K_{2}\right)=\frac{1}{2} \sum_{p} \varepsilon(p) .
$$

It is easy to check that $w\left(K_{1}, K_{2}\right)$ is invariant under the Reidemeister moves and hence is a link invariant. As an example, the linking number of the strands in $\widetilde{U}$ in Figure 1.1 is $f=3$.

Once we specify $K$ and the linking number $f$, this uniquely determines $\widetilde{K}$. Therefore it is natural to think of $K$ as a framed knot, and we will do so from now on. We will always assume that the framing is equal to the linking number of the strands in $\widetilde{K}$.

We can now state our first main theorem.

Theorem 1. Let $K$ be an oriented, framed knot, with framing number $f$. Let $\widetilde{K}$ be the two-component link obtained as described above. Then

$$
t^{f}\left(1+t+t^{-1}\right) F_{K}\left(i t^{-2}, i\left(t-t^{-1}\right)\right)=-\left(t^{1 / 2}+t^{-1 / 2}\right) V_{\widetilde{K}}(t)-t^{3 f} .
$$

The proof of Theorem 1 is given in $\S 3$. Before proceeding, we present some examples which test (1.3). First of all, when $K$ is the unknot $U$, we have the situation depicted in Figure 1.1. It is easy to compute the Jones polynomial for 

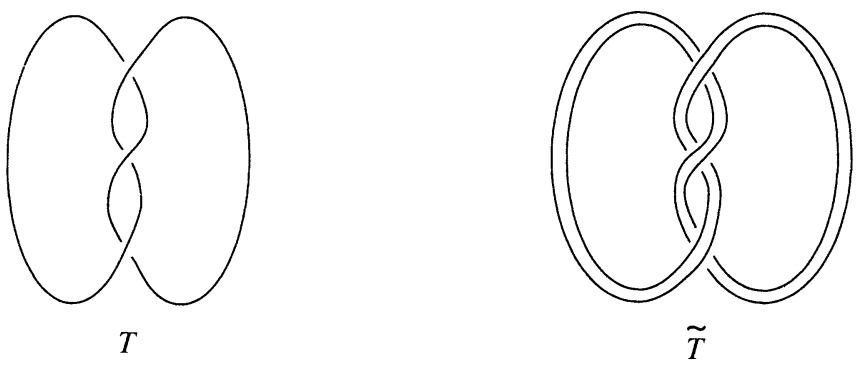

Figure 1.3. The trefoil and a derived link
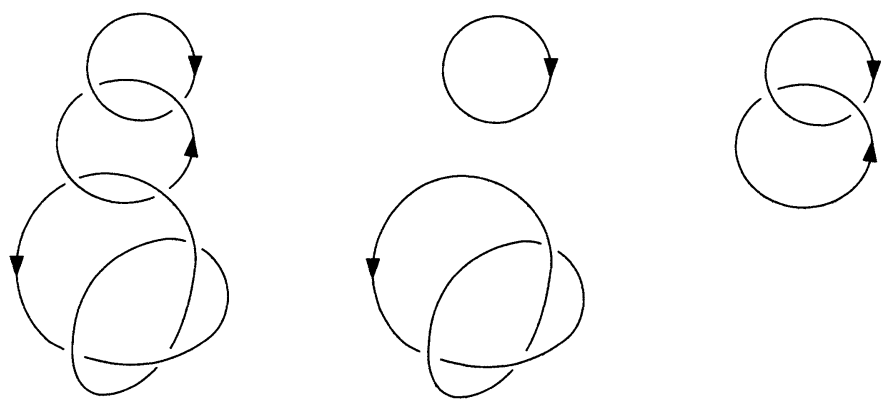

Figure 1.4. A link and two of its sublinks

$\widetilde{U}$ with an arbitrary framing $f$, and it is given by

$$
V_{\widetilde{U}}(t)=-\left(t^{1 / 2}+t^{-1 / 2}\right)^{-1}\left(t^{3 f}+t^{f+1}+t^{f}+t^{f-1}\right) .
$$

Since $U$ is the unknot, $F_{U}=1$, and we see that (1.3) is verified.

A more formidable test is provided by the example in Figure 1.3, where $T$ is the trefoil. In this case, the Kauffman polynomial of $K$ is easily computed, and we obtain

$$
F_{T}\left(i t^{-2}, i\left(t-t^{-1}\right)\right)=t^{11}-t^{10}-t^{9}+t^{8}-t^{7}+t^{5}+t^{2} .
$$

The Jones polynomial of $\widetilde{T}$ can be computed with some difficulty, and we obtain

$$
-\left(t^{1 / 2}+t^{-1 / 2}\right) V_{\widetilde{T}}(t)=t^{15}-t^{13}-t^{12}-t^{11}+t^{9}+t^{8}+t^{7}+t^{6}+t^{5}+t^{4}
$$

From Figure 1.3 we can read off the linking number and we get $f=3$. The reader can check that (1.3) is satisfied when (1.5) and (1.6) are substituted in.

We will now describe the corresponding result for a general framed link $L$. Once again, we want to separate each component of $L$ into two parallel strands. So we will need to specify an integer for each component of $L$, which will equal the linking number of the two strands in that component. This is equivalent to saying that $L$ is a framed link.

Suppose the components of $L$ are $\left\{C_{1}, \ldots, C_{n}\right\}$, and $f\left(C_{k}\right)$ is the framing of the component $C_{k}$. We will write $w\left(C_{k}, C_{l}\right)$ for the linking number of the components $C_{k}$ and $C_{l}$. Furthermore, we will want to consider sublinks of $L$, obtained by removing from $L$ some of its components, without disturbing the others. Some examples are shown in Figure 1.4. 
Given a subset $J$ of $\left\{C_{1}, \ldots, C_{n}\right\}$, we will write $J \subset L$ to indicate that $J$ is a sublink of $L$. Given a sublink $J$, we define

$$
\begin{gathered}
f(J)=\sum_{k: C_{k} \in J} f\left(C_{k}\right), \\
n(J)=\sum_{k, l: C_{k} \in J} w\left(C_{k}, C_{l}\right) .
\end{gathered}
$$

We want to allow $J$ to be the empty set, in which case (1.7) and (1.8) are zero. As usual, we will denote by $|J|$ the cardinality of $J$, and by $J^{c}$ the complement of $J$. Finally, we adopt the convention that when $J=\varnothing$ is the empty set

$$
V_{\varnothing}(t)=-\left(t^{1 / 2}+t^{-1 / 2}\right)^{-1} .
$$

Theorem 2. Let $L$ be a framed, oriented link. Then with the notation and conventions defined above.

$$
\begin{aligned}
& \left(1+t+t^{-1}\right) t^{f(L)-4 n(L)} F_{L}\left(i t^{-2}, i\left(t-t^{-1}\right)\right) \\
& \quad=\left(t^{1 / 2}+t^{-1 / 2}\right) \sum_{J \subset L}(-1)^{|J|} t^{3 f\left(J^{c}\right)-6 n(J)} V_{\widetilde{J}}(t) .
\end{aligned}
$$

This result will be proved in $\S 3$. Notice that if $L$ has $n$ components, the right-hand side of (1.10) has $2^{n}$ terms. Also when $n$ is one, Theorem 2 reduces to Theorem 1.

Once again, we present some test examples. When $L$ is composed of $k$ unlinked unknots, each with zero framing, the left-hand side of (1.10) is

$$
(-1)^{k-1}\left(1+t+t^{-1}\right)^{k} \text {. }
$$

The right-hand side of $(1.10)$ is

$$
\begin{aligned}
\left(t^{1 / 2}\right. & \left.+t^{-1 / 2}\right) \sum_{J \subset L}(-1)^{|J|}(-1)\left(t^{1 / 2}+t^{-1 / 2}\right)^{-1}\left(2+t+t^{-1}\right)^{|J|} \\
& =(-1)^{k+1} \sum_{J \subset L}(-1)^{\left|J^{c}\right|}\left(2+t+t^{-1}\right)^{|J|} \\
& =(-1)^{k+1}\left(2+t+t^{-1}-1\right)^{k} .
\end{aligned}
$$

A less trivial example is provided by the two-component link in Figure 1.5. We denote by $C_{1}$ and $C_{2}$ the left- and right-hand components of $L$, and then we have

$$
f\left(C_{1}\right)=f\left(C_{2}\right)=0, \quad w\left(C_{1}, C_{2}\right)=1 .
$$

The left-hand side of (1.10) is easily computed, and we get

$$
\left(1+t+t^{-1}\right) t^{-4} F_{L}\left(i t^{-2}, i\left(t-t^{-1}\right)\right)=-\left(1+t+t^{-1}\right) t^{-4}\left(t^{7}+t^{4}+t\right) .
$$

The right-hand side of (1.10) has four terms

$$
\left(t^{1 / 2}+t^{-1 / 2}\right)\left\{t^{-6} V_{\widetilde{L}}(t)-V_{\widetilde{C}_{1}}(t)-V_{\widetilde{C}_{2}}(t)+V_{\varnothing}(t)\right\} .
$$




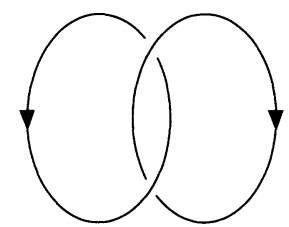

$L$

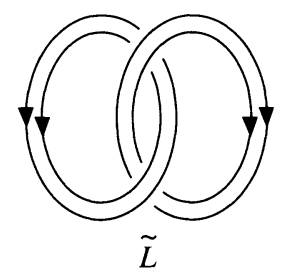

FIGURE 1.5. Two linked circles and a derived link

By explicit computation, we get

$$
\begin{gathered}
\left(t^{1 / 2}+t^{-1 / 2}\right) V_{\widetilde{L}}(t)=-\left(t^{10}+t^{9}+t^{8}+3 t^{7}+4 t^{6}+3 t^{5}+t^{4}+t^{3}+t^{2}\right), \\
V_{\widetilde{C}_{1}}(t)=V_{\widetilde{C}_{2}}(t)=-\left(t^{1 / 2}+t^{-1 / 2}\right), \quad V_{\varnothing}(t)=-\left(t^{1 / 2}+t^{-1 / 2}\right)^{-1}
\end{gathered}
$$

The reader can check that when (1.16) is substituted into (1.15), we reproduce (1.14).

In terms of utility, Theorem 2 is back to front. It expresses a specialization of the Kauffman polynomial of a link $L$ in terms of the Jones polynomials of more complicated links. However, the relation can be inverted to express $V_{\widetilde{L}}(t)$ in terms of the Kauffman polynomials of $L$, and its sublinks. This time we use the convention that

$$
F_{\varnothing}\left(i t^{-2}, i\left(t-t^{-1}\right)\right)=-\left(1+t+t^{-1}\right)^{-1}
$$

Theorem 3.

$$
\begin{aligned}
\left(t^{1 / 2}+t^{-1 / 2}\right) t^{-6 n(L)} V_{\widetilde{L}}(t) \\
=\left(1+t+t^{-1}\right) \sum_{J \subset L}(-1)^{|J|} t^{f(J)+3 f\left(J^{c}\right)-4 n(J)} F_{J}\left(i t^{-2}, i\left(t-t^{-1}\right)\right) .
\end{aligned}
$$

The proofs of Theorems 1, 2, 3 are presented in $\S 3$. They are completely elementary in the sense that they use only the crossing relations which serve as definitions of the Jones and Kauffman polynomials. A good review of these relations can be found in $[2,4]$.

The author was led to search for relations of this kind after studying the representations of the braid group which lead to link invariants [5-9]. In particular, these representations arise in conformal field theory $[10,11]$. The idea of "fusion" in conformal field theory is closely related to the process of going from a two-stranded link $\widetilde{K}$ to a knot $K$, as described at the beginning of this section.

\section{CROSSING RELATIONS FOR THE JONES POLYNOMIAL}

In this section we derive new crossing relations for the Jones polynomial. These are the essential ingredients in the proof of Theorem 2 .

We begin by recalling the usual crossing relations for the Jones polynomial. These include the original relation stated by Jones [1], as well as later additions by Birman and Kanenobu [12]. Suppose three links $L_{+}, L_{-}$and $L_{0}$ differ only at one crossing, in the manner indicated in Figure 2.1. Then the corresponding link polynomials satisfy the relation

$$
V_{L_{+}}=t^{2} V_{L_{-}}-t^{1 / 2}(1-t) V_{L_{0}} .
$$



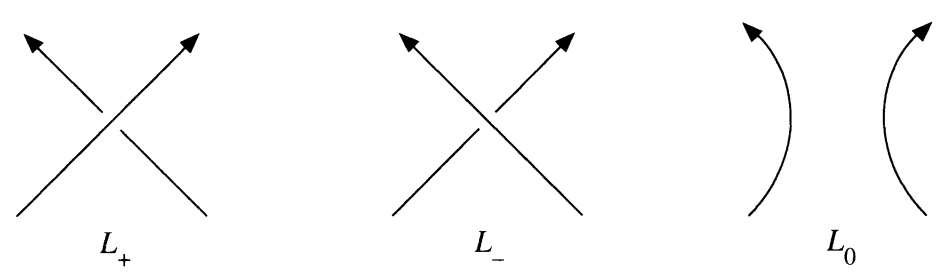

FIGURE 2.1. Crossings for the Jones polynomial

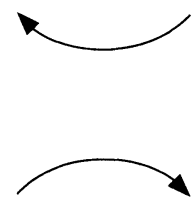

(a)

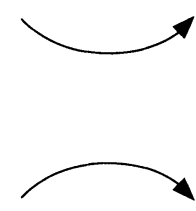

(b)

Figure 2.2. $L_{\infty}$ for (a) Case 1 and (b) Case 2

Together with the relation $V_{U}=1$ for the unknot $U$, this suffices to determine $V_{L}(t)$ for any link $L$. As Birman pointed out, there is another relation satisfied by $V_{L}(t)$. This divides into two cases, as follows.

Case 1. The two links in $L_{+}$shown in Figure 2.1 belong to the same component of $L_{+}$. In this case, we introduce a new link $L_{\infty}$ as shown in Figure 2.2(a).

Notice that the orientation of some links have been changed. Also the two lines in $L_{0}$ shown in Figure 2.1 belong to different components now. Let $\lambda$ be the linking number of the right-hand component with the rest of $L_{0}$. Then

$$
V_{L_{+}}=t V_{L_{-}}+(1-t) t^{3 \lambda} V_{L_{\infty}} \text {. }
$$

Case 2. The two lines in $L_{+}$shown in Figure 2.1 belong to different components. In this case, we define the link $L_{\infty}$ as indicated in Figure 2.2(b). Also, let $\mu$ be the linking number of the bottom right to top left component of $L_{+}$ shown in Figure 2.1 with the rest of $L_{+}$. Then

$$
V_{L_{+}}=t V_{L_{-}}+(1-t) t^{3(\mu-1 / 2)} V_{L_{\infty}} .
$$
1.

Using (2.1) and (2.2), we can deduce the following further relations for Case

$$
V_{L_{+}}=-t^{1 / 2} V_{L_{0}}-t^{3 \lambda+1} V_{L_{\infty}}, \quad V_{L_{-}}=-t^{-1 / 2} V_{L_{0}}-t^{3 \lambda-1} V_{L_{\infty}}
$$

Similarly in Case 2 we have

$$
V_{L_{+}}=-t^{1 / 2} V_{L_{0}}-t^{3 \mu-1 / 2} V_{L_{\infty}}, \quad V_{L_{-}}=-t^{-1 / 2} V_{L_{0}}-t^{3 \mu-5 / 2} V_{L_{\infty}} .
$$

Our new relations will concern crossings of pairs of lines, which arise when a link $L$ is replaced by $\widetilde{L}$. The diagrams corresponding to Figure 2.1 are shown in Figure 2.3. The relations satisfied by $V_{\widetilde{L}_{+}}(t)$ and $V_{\widetilde{L}_{-}}(t)$ separate into the two cases described previously. We will state and prove the result for Case 1 first, and then the result for Case 2. 

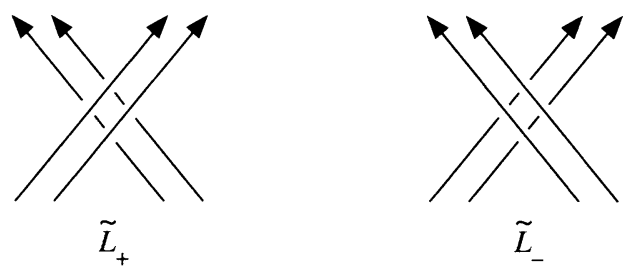

FIGURE 2.3. Crossings in $\widetilde{L}$
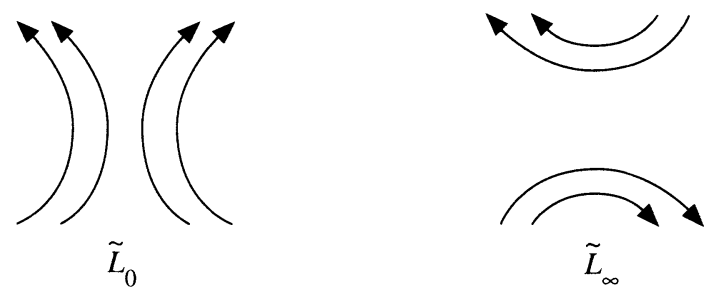

Figure 2.4. Crossings in $\widetilde{L}_{0}, \widetilde{L}_{\infty}$ for Case 1

Case 1. In order to simplify the notation, we will let $C_{+}$denote the component of $L_{+}$which contains the line segments shown in Figure 2.1, and let $M$ denote the remainder of $L_{+}$. This allows us to write $L_{+}=M \cup C_{+}$. Similarly we write

$$
L_{-}=M \cup C_{-}, \quad L_{\infty}=M \cup C_{\infty} .
$$

The link $L_{0}$ has two components corresponding to $C_{+}$. We call these $C_{L}$ and $C_{R}$, for the left- and right-hand sides shown in Figure 2.1. Then

$$
L_{0}=M \cup C_{L} \cup C_{R} .
$$

The corresponding crossings in $\widetilde{L}_{0}$ and $\widetilde{L}_{\infty}$ are shown in Figure 2.4. In addition, we will also encounter links in which one of the components $C_{L}$ or $C_{R}$ has been removed. The doubled links will be denoted $\widetilde{L}_{L}$ and $\widetilde{L}_{R}$ respectively. When both components $C_{L}$ and $C_{R}$ are removed, the doubled link is just $\widetilde{M}$.

In order to state our results, we also need to specify some linking numbers and framings. We define

$$
\begin{gathered}
\alpha=w\left(C_{L}, C_{R}\right), \\
w_{L}(M)=w\left(C_{L}, M\right)=\sum_{k: C_{k} \in M} w\left(C_{L}, C_{k}\right), \\
w_{R}(M)=w\left(C_{R}, M\right) .
\end{gathered}
$$

Also we define the framings on $C_{L}$ and $C_{R}$ to be

$$
f\left(C_{L}\right)=f_{L}, \quad f\left(C_{R}\right)=f_{R} .
$$

Using these values, we can compute the framings of $C_{+}, C_{-}$and $C_{\infty}$. The results are

$$
\begin{gathered}
f\left(C_{+}\right)=f_{L}+f_{R}+2 \alpha+1, \quad f\left(C_{-}\right)=f_{L}+f_{R}+2 \alpha-1, \\
f\left(C_{\infty}\right)=f_{L}+f_{R}-2 \alpha .
\end{gathered}
$$



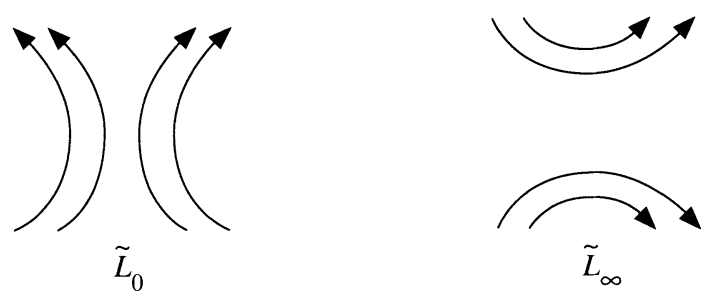

FiguRE 2.5. Crossings in $\widetilde{L}_{0}, \widetilde{L}_{\infty}$ for Case 2

Our first result is the following:

Proposition 2.1.

(2.9)

$$
\begin{aligned}
& t^{-3} V_{\widetilde{L}_{+}}(t)-t^{3} V_{\widetilde{L}_{-}}(t) \\
& =\left(t-t^{-1}\right)\left\{t^{12 \alpha+12 w_{R}(M)} V_{\widetilde{L}_{\infty}}(t)-V_{\widetilde{L}_{0}}(t)+t^{3 f_{R}+6 \alpha+6 w_{R}(M)} V_{\widetilde{L}_{L}}(t)\right. \\
& \left.+t^{3 f_{L}+6 \alpha+6 w_{L}(M)} V_{\widetilde{L}_{R}}(t)-2 t^{3 f_{L}+3 f_{R}+6 \alpha+6 w_{L}(M)+6 w_{R}(M)} V_{\widetilde{M}}(t)\right\} .
\end{aligned}
$$

Proof. The proof follows by a sequence of applications of the crossing relations (2.1)-(2.5). We will indicate the steps in the computation by using diagrams to represent link polynomials. These diagrams will be called $D_{1}, D_{2}, \ldots, D_{15}$ in the following equations. They are shown in Figure 2.6. First we apply (2.1) and $(2.2)$ to $V_{\widetilde{L}_{+}}(t)$ and $V_{\widetilde{L}_{-}}(t)$.

$$
\begin{aligned}
D_{1}= & t^{3} D_{2}+(1-t) t^{7 / 2} t^{3 f_{L}+3 f_{R}+6 \alpha+6 w_{L}(M)+6 w_{R}(M)} D_{3} \\
& -(1-t) t^{1 / 2} D_{4} . \\
D_{5}= & t^{-3} D_{2}-(1-t) t^{-9 / 2} t^{3 f_{L}+3 f_{R}+6 \alpha+6 w_{L}(M)+6 w_{R}(M)} D_{6} \\
& +(1-t) t^{-3 / 2} D_{7} .
\end{aligned}
$$

Combining the expressions in (2.10) and (2.11) we get

$$
\begin{aligned}
& t^{-3} V_{\widetilde{L}_{+}(t)-t^{3} V_{L_{-}}}(t)=(1-t) t^{1 / 2} t^{3 f_{L}+3 f_{R}+6 \alpha+6 w_{L}(M)+6 w_{R}(M)} D_{3} \\
& \quad+(1-t) t^{-3 / 2} t^{3 f_{L}+3 f_{R}+6 \alpha+6 w_{L}(M)+6 w_{R}(M)} D_{6} \\
& \quad-(1-t) t^{-5 / 2} D_{4}-(1-t) t^{3 / 2} D_{7} .
\end{aligned}
$$

Now we use (2.4) twice on each term in (2.12). As an example, we write out the result of this for the first diagram on the right-hand side of (2.12).

$$
\begin{gathered}
D_{3}=t^{-3 / 2} t^{-3 f_{L}-6 w_{L}(M)} D_{8}-t^{-1 / 2} t^{-3 f_{L}-6 w_{L}(M)} D_{9} \\
-t^{-3 / 2} t^{-3 f_{R}-6 w_{R}(M)} D_{10}+t^{-3 / 2}(1+t) .
\end{gathered}
$$

In the second term on the right side of (2.13), the component $C_{R}$ has been removed completely from the link. The diagram therefore represents $V_{\widetilde{L}_{L}}(t)$. Similarly $C_{L}$ has been removed in the third term, and both $C_{L}$ and $C_{R}$ have been removed in the fourth term. The corresponding polynomials are $V_{\widetilde{L}_{R}}(t)$ and $V_{\widetilde{M}}(t)$, respectively. 


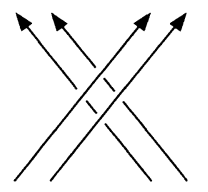

$D_{1}$

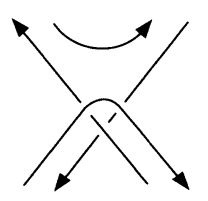

$D_{6}$

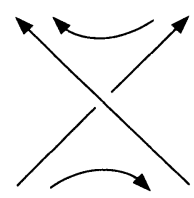

$D_{11}$

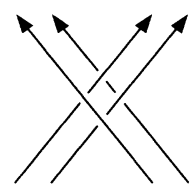

$\mathrm{D}_{2}$

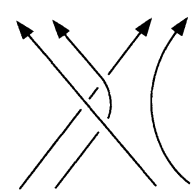

$D_{7}$

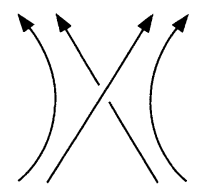

$D_{12}$

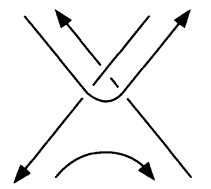

$D_{3}$

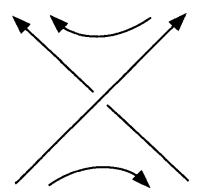

$D_{8}$

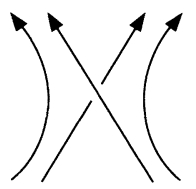

$D_{13}$

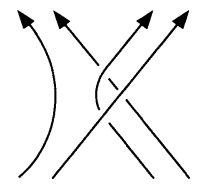

$D_{4}$

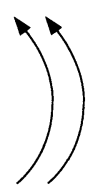

$D_{9}$

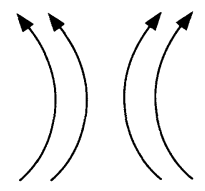

$D_{14}$

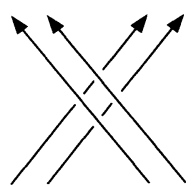

$D_{5}$

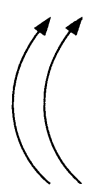

$D_{10}$

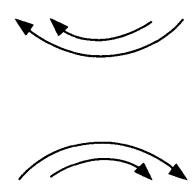

$D_{15}$

FIGURE 2.6. Diagrams for link polynomials in Case 1

Performing the same expansion for the other terms in (2.12) and combining the results, we get the following:

(2.14)

$$
\begin{aligned}
t^{-3} V_{\widetilde{L}_{+}} & (t)-t^{3} V_{\widetilde{L}_{-}}(t) \\
= & \left(t-t^{-1}\right)\left\{t^{3 f_{R}+6 \alpha+6 w_{R}(M)} V_{\widetilde{L}_{L}}(t)+t^{3 f_{L}+6 \alpha+6 w_{L}(M)} V_{\widetilde{L}_{R}}(t)\right\} \\
& +(1-t) t^{-1 / 2}\left\{t^{3 f_{R}+6 \alpha+6 w_{R}(M)}\left(t^{-1 / 2} D_{8}+t^{1 / 2} D_{11}\right)-\left(t^{-1} D_{12}+t D_{13}\right)\right\} \\
& -2\left(t-t^{-1}\right) t^{3 f_{L}+3 f_{R}+6 \alpha+6 w_{L}(M)+6 w_{R}(M)} V_{\widetilde{M}}(t) .
\end{aligned}
$$

A final application of (2.4) gives the following identity:

$$
\begin{gathered}
t^{3 f_{R}+6 \alpha+6 w_{R}(M)}\left(t^{-1 / 2} D_{8}+t^{1 / 2} D_{11}\right)-\left(t^{-1} D_{12}+t D_{13}\right) \\
=\left(t^{1 / 2}+t^{-1 / 2}\right)\left(D_{14}-t^{12 \alpha+12 w_{R}(M)} D_{15}\right) .
\end{gathered}
$$

The first and second diagrams on the right side of (2.15) represent $V_{\widetilde{L}_{0}}$ and $V_{\widetilde{L}_{\infty}}$ respectively. Substituting (2.15) into (2.14), we obtain the result (2.9). Q.E.D.

Case 2. In this case, the link $L_{+}$shown in Figure 2.1 has two components displayed. We will denote by $C_{1}$ the component going from the bottom right to the top left, and by $C_{2}$ the other component. Once again, the remainder of $L_{+}$is denoted by $M$, so we have

$$
L_{+}=M \cup C_{1} \cup C_{2} .
$$



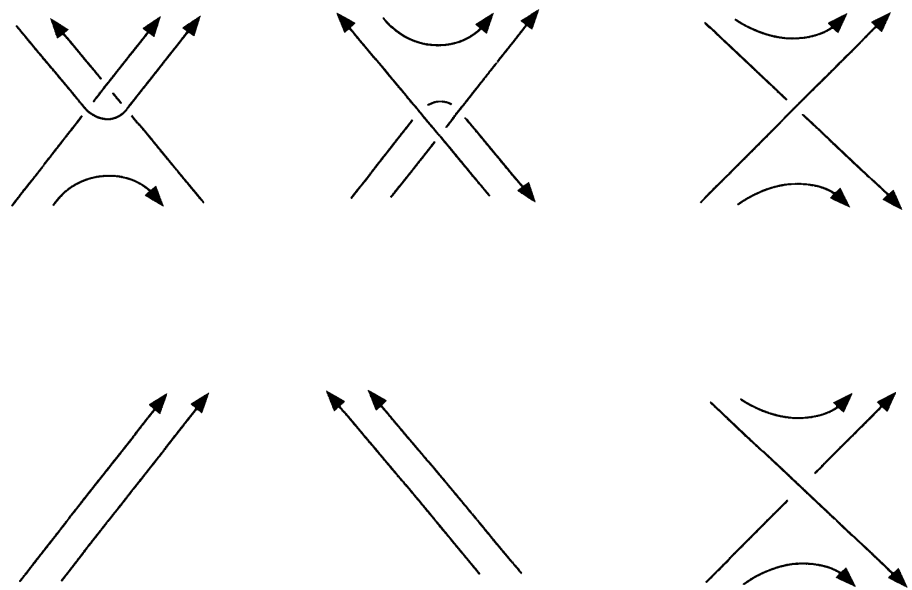

FIGURE 2.7. Diagrams for link polynomials in Case 2

The doubled links $\widetilde{L}_{0}$ and $\widetilde{L}_{\infty}$ are shown in Figure 2.5. We will need the following linking numbers and framings:

$$
\begin{gathered}
\beta=w\left(C_{1}, C_{2}\right), \\
w_{1}(M)=w\left(C_{1}, M\right), \quad w_{2}(M)=w\left(C_{2}, M\right), \\
f_{1}=f\left(C_{1}\right), \quad f_{2}=f\left(C_{2}\right) .
\end{gathered}
$$

Computing the framings in $C_{0}$ and $C_{\infty}$, we get

$$
f\left(C_{0}\right)=f_{1}+f_{2}+2 \beta-1, \quad f\left(C_{\infty}\right)=f_{1}+f_{2}-2 \beta+1 .
$$

We now have our second result.

\section{Proposition 2.2.}

$$
t^{-3} V_{\widetilde{L}_{+}}(t)-t^{3} V_{\widetilde{L}_{-}}(t)=\left(t-t^{-1}\right)\left\{t^{12 \beta-6+12 w_{1}(M)} V_{\widetilde{L}_{\infty}}(t)-V_{\widetilde{L}_{0}}(t)\right\}
$$

Proof. Once again, the proof involves application of the identities (2.1), (2.3), (2.5). We present the main steps in the computation below. There are some new diagrams $D_{16}, \ldots, D_{21}$ which are shown in Figure 2.7.

$$
\begin{aligned}
& D_{1}=t^{3} D_{2}+(1-t) t^{-5 / 2} t^{3 f_{1}+6 \beta+6 w_{1}(M)} D_{16}-(1-t) t^{1 / 2} D_{4} \\
& D_{5}=t^{-3} D_{2}-(1-t) t^{-9 / 2} t^{3 f_{1}+6 \beta+6 w_{1}(M)} D_{17}+(1-t) t^{-3 / 2} D_{7} .
\end{aligned}
$$

As an example, we give the expansion of the second term in (2.19) below.

$$
\begin{aligned}
D_{16}= & t^{-3 f_{1}+6 \beta+6 w_{1}(M)} D_{18}-t^{3 / 2} D_{19} \\
& -t^{5 / 2} t^{-3 f_{1}+3 f_{2}-6 w_{1}(M)+6 w_{2}(M)} D_{20}+t^{3 / 2}(1+t) t^{3 f_{2}+6 w_{2}(M)} .
\end{aligned}
$$

In the second term on the right in (2.21), the component $C_{1}$ has been removed from $L_{+}$. Similarly in the third term, $C_{2}$ has been removed, and both $C_{1}$ and $C_{2}$ are absent in the fourth term.

Making similar expansions for the other terms in (2.19) and (2.20), we deduce the following relation:

$$
\begin{array}{r}
t^{-3} V_{\widetilde{L}_{+}}(t)-t^{3} V_{\widetilde{L}_{-}}(t)=(1-t) t^{-1 / 2}\left\{t^{12 \beta-6+12 w_{1}(M)}\left(t^{-1} D_{21}+t D_{18}\right)\right. \\
\left.-\left(t^{-1} D_{12}+t D_{13}\right)\right\}
\end{array}
$$


One last application of (2.5) to each term in (2.22) produces the desired result (2.18). Q.E.D.

\section{PROOFS OF THEOREMS}

Since Theorem 1 is a special case of Theorem 2, we need only establish the result stated in Theorem 2 . We do this by using the results from $\S 2$ to analyze the right-hand side of (1.10), and prove that it satisfies the crossing relations of the Kauffman polynomial. In $\S 2$, we stated the usual crossing relations for the Jones polynomial. The corresponding relations for the Kauffman polynomial separate into the same two cases [3].

Case 1. With the same notation as in $\S 2$,

$$
a F_{L_{+}}(a, x)+a^{-1} F_{L_{-}}(a, x)=x\left\{F_{L_{0}}(a, x)+a^{-4 \lambda} F_{L_{\infty}}(a, x)\right\} .
$$

Case 2. Again with the same notation,

$$
a F_{L_{+}}(a, x)+a^{-1} F_{L_{-}}(a, x)=x\left\{F_{L_{0}}(a, x)+a^{-4 \mu+2} F_{L_{\infty}}(a, x)\right\} .
$$

As mentioned before, we will prove Theorem 2 by showing that the expression (1.10) satisfies the relations (3.1) and (3.2) which define the Kauffman polynomial. As a first step, we will show that the expression (1.10) transforms correctly when the framing of the link is changed. For this, it is sufficient to consider what happens when the framing of one component in $L$ changes by one. We will list the components of $L$ as $\left\{C_{1}, \ldots, C_{n}\right\}$, and then $f\left(C_{k}\right)$ is the framing of the component $C_{k}$. Also $w\left(C_{k}, C_{l}\right)$ is the linking number of components $C_{k}$ and $C_{l}$. If $M$ is a sublink of $L$ which does not contain $C_{k}$, we will write

$$
w\left(C_{k}, M\right)=\sum_{l: C_{l} \in M} w\left(C_{k}, C_{l}\right) .
$$

Suppose now that we single out one component $C_{k}$ whose framing we want to change. Then we can rewrite the right-hand side of (1.10) as follows:

$$
\begin{aligned}
&\left(t^{1 / 2}+t^{-1 / 2}\right) \sum_{J \subset L}(-1)^{|J|} t^{3 f\left(J^{c}\right)-6 n(J)} V_{\widetilde{J}}(t) \\
&=\left(t^{1 / 2}+t^{-1 / 2}\right) \sum_{M \subset L \backslash C_{k}}(-1)^{|M|}\left\{t^{3 f(\backslash M)-6 n(M)} V_{\widetilde{M}}(t)\right.\left.-t^{3 f(L \backslash M)-3 f\left(C_{k}\right)-6 n\left(M \cup C_{k}\right)} V_{\widetilde{M} \cup \widetilde{C}_{k}}(t)\right\} \\
&=\left(t^{1 / 2}+t^{-1 / 2}\right) \sum_{M \subset L \backslash C_{k}}(-1)^{|M|} t^{3 f(L \backslash M)-6 n(M)} \\
& \quad \times\left\{V_{\widetilde{M}}(t)-t^{-3 f\left(C_{k}\right)-6 w\left(C_{k}, M\right)} V_{\widetilde{M} \cup \widetilde{C}_{k}}(t)\right\} .
\end{aligned}
$$

Now we can use the relation (2.2) to see what happens when the framing on $C_{k}$ is changed. Suppose that the two strands in $\widetilde{C}_{k}$ are twisted as shown in Figure 3.1(a). 


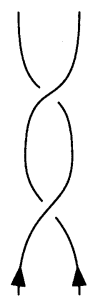

(a)

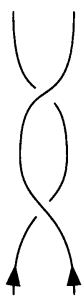

(b)

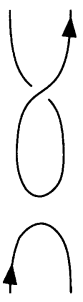

(c)

FIGURE 3.1. Changing framing by one

By applying (2.2), we get a relation between the Jones polynomials of the three links shown in Figure 3.1. Clearly the framing in (b) is one less than in (a). Also, since $\widetilde{C}_{k}$ is composed of two parallel strands, the diagram in (c) collapses down to the unknot. Let us denote by $\widetilde{C}_{k}^{\prime}$ the two strands shown in Figure 3.1(b), whose linking number is $f\left(C_{k}\right)-1$. Applying (2.2) gives

$$
V_{\widetilde{M} \cup \widetilde{C}_{k}}(t)=t V_{\widetilde{M} \cup \widetilde{C}_{k}^{\prime}}(t)+\left(1-t^{-2}\right) t^{3 f\left(C_{k}\right)+6 w\left(C_{k}, M\right)} V_{\widetilde{M}}(t) .
$$

Substituting (3.5) into (3.4) gives

$$
\begin{aligned}
\left(t^{1 / 2}+t^{-1 / 2}\right) t \sum_{M \subset L \backslash C_{k}}( & -1)^{|M|} t^{3 f(L \backslash M)-6 n(M)} \\
& \times\left\{V_{\widetilde{M}}(t)-t^{-3\left[f\left(C_{k}\right)-1\right]-6 w\left(C_{k}, M\right)} V_{\widetilde{M} \cup{\widetilde{C_{k}^{\prime}}}_{k}^{\prime}}(t)\right\} .
\end{aligned}
$$

Apart from the overall factor of $t,(3.6)$ is the right-hand side of (1.10) corresponding to the framed link $L$ with the framing of $C_{k}$ reduced by one. Furthermore, the left-hand side of (1.10) also changes by a factor of $t$ when $f\left(C_{k}\right)$ is reduced by one. Therefore if $(1.10)$ holds for any particular choice of framing, it also holds for all other framings.

We now turn to the proof of Theorem 2. We will choose a particular framing which simplifies the proof, and then appeal to the previous argument to derive the general result. We will consider separately the two cases distinguished earlier.

Case 1. We will use the same notation introduced for Case 1 in $\S 2$. We choose the framings $f_{L}$ and $f_{R}$ to be zero, and also choose each component of $M$ to have zero framing. We can now compute the following relations between linking numbers:

$$
\begin{gathered}
n\left(L_{+}\right)=n\left(L_{-}\right)=n(M)+w_{L}(M)+w_{R}(M), \\
n\left(L_{0}\right)=n(M)+w_{L}(M)+w_{R}(M)+\alpha, \\
n\left(L_{\infty}\right)=n(M)+w_{L}(M)-w_{R}(M) .
\end{gathered}
$$

Similar relations hold if $M$ is replaced by any sublink $J$ of $M$, and if $L_{+}$ is replaced by $J \cup C_{+}$, etc. We will now write down the four expressions which (1.10) provides for the four links $L_{+}, L_{-}, L_{0}$ and $L_{\infty}$. We again collect 
terms together in the manner of (3.4). The left-hand side will be denoted $F_{+}$, $F_{-}, F_{0}$ and $F_{\infty}$ respectively. For convenience, we will write $g$ instead of $\left(t^{1 / 2}+t^{-1 / 2}\right)\left(1+t+t^{-1}\right)^{-1}$.

$\left(\mathrm{A}_{1}\right)$

$$
\begin{aligned}
& t^{1+2 \alpha-4 n(M)-4 w_{L}(M)-4 w_{R}(M)} F_{+} \\
& =g \sum_{J \subset M}(-1)^{|J|}\left\{t^{3+6 \alpha-6 n(J)} V_{\widetilde{J}}(t)\right. \\
& \left.\quad-t^{-6 n(J)-6 w_{L}(J)-6 w_{R}(J)} V_{\widetilde{J} \cup \widetilde{C}_{+}}(t)\right\} .
\end{aligned}
$$

$$
t^{-1+2 \alpha-4 n(M)-4 w_{L}(M)-4 w_{R}(M)} F_{-}
$$

$\left(\mathrm{A}_{2}\right)$

$$
\begin{aligned}
=g \sum_{J \subset M}(-1)^{|J|}\left\{t^{-3+6 \alpha-6 n(J)} V_{\widetilde{J}}(t)\right. \\
-t^{-6 n(J)-6 w_{L}(J)-6 w_{R}(J)} V_{\left.\widetilde{J} \cup \widetilde{C}_{-}(t)\right\} .}
\end{aligned}
$$

$\left(\mathrm{A}_{3}\right)$

$$
\begin{aligned}
& t^{-4 n(M)-4 w_{L}(M)-4 w_{R}(M)-4 \alpha} F_{0} \\
&=g \sum_{J \subset M}(-1)^{|J|}\left\{t^{-6 n(J)} V_{\widetilde{J}}(t)-t^{-6 n(J)-6 w_{L}(J)} V_{\widetilde{J} \cup \widetilde{C}_{L}}(t)\right. \\
&-t^{-6 n(J)-6 w_{R}(J)} V_{\widetilde{J} \cup \widetilde{C}_{R}}(t) \\
&+t^{-6 n(J)-6 w_{L}(J)-6 w_{R}(J)-6 \alpha} V_{\left.\widetilde{J} \cup \widetilde{C}_{L} \cup \widetilde{C}_{R}(t)\right\} .}
\end{aligned}
$$

$$
\begin{aligned}
& t^{-2 \alpha-4 n(M)-4 w_{L}(M)+4 w_{R}(M)} F_{\infty} \\
& =g \sum_{J \subset M}(-1)^{|J|}\left\{t^{-6 \alpha-6 n(J)} V_{\widetilde{J}}(t)\right. \\
& \quad-t^{-6 n(J)-6 w_{L}(J)+6 w_{R}(J)} V_{\left.\widetilde{J} \cup \widetilde{C}_{\infty}(t)\right\} .}
\end{aligned}
$$

Our strategy now is to show that $F_{+}, F_{-}, F_{0}$ and $F_{\infty}$ satisfy the crossing relation (3.1). In order to do this, we consider the following combination of expressions $\left(A_{1}\right)$ and $\left(A_{2}\right)$ :

$$
\begin{aligned}
t^{-3} A_{1}-t^{3} A_{2}=-g \sum_{J \subset M}( & -1)^{|J|} t^{-6 n(J)-6 w_{L}(J)-6 w_{R}(J)} \\
& \times\left\{t^{-3} V_{\widetilde{J} \cup \widetilde{C}_{+}}(t)-t^{3} V_{\widetilde{J} \cup \widetilde{C}_{-}}(t)\right\} .
\end{aligned}
$$

Now we use the result of Proposition 2.1, applying it to $J \cup C_{+}$and $J \cup C_{-}$. Recall that $f_{L}$ and $f_{R}$ are zero.

$$
\begin{aligned}
& t^{-3} A_{1}-t^{3} A_{2}=\left(t-t^{-1}\right) g \sum_{J \subset M}(-1)^{|J|} t^{-6 n(J)-6 w_{L}(J)-6 w_{R}(J)}
\end{aligned}
$$

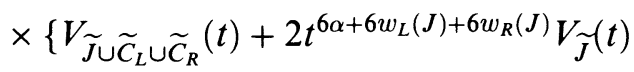

$$
\begin{aligned}
& -t^{12 \alpha+12 w_{R}(J)} V_{\widetilde{J} \cup \widetilde{C}_{\infty}}(t)-t^{6 \alpha+6 w_{R}(J)} V_{\widetilde{J} \cup \widetilde{C}_{L}}(t) \\
& \left.-t^{6 \alpha+6 w_{L}(J)} V_{\widetilde{J} \cup \widetilde{C}_{R}}(t)\right\} .
\end{aligned}
$$


Combining expressions $\left(\mathrm{A}_{3}\right)$ and $\left(\mathrm{A}_{4}\right)$, we also have

$$
\begin{aligned}
t^{6 \alpha} A_{3}+ & t^{12 \alpha} A_{4} \\
=g \sum_{J \subset M}(-1)^{|J|} t^{-6 n(J)}\{ & 2 t^{6 \alpha} V_{\widetilde{J}}(t)-t^{6 \alpha-6 w_{L}(J)} V_{\widetilde{J} \cup \widetilde{C}_{L}}(t) \\
& -t^{6 \alpha-6 w_{R}(J)} V_{\widetilde{J} \cup \widetilde{C}_{R}}(t) \\
& +t^{-6 w_{L}(J)-6 w_{R}(J)} V_{\widetilde{J} \cup \widetilde{C}_{L} \cup \widetilde{C}_{R}}(t) \\
& -t^{12 \alpha-6 w_{L}(J)+6 w_{R}(J)} V_{\left.\widetilde{J} \cup \widetilde{C}_{\infty}(t)\right\} .}
\end{aligned}
$$

Combining (3.9) and (3.10), we deduce that

$$
t^{-3} A_{1}-t^{3} A_{2}=\left(t-t^{-1}\right)\left(t^{6 \alpha} A_{3}+t^{12 \alpha} A_{4}\right)
$$

Inserting the left sides of the expressions into (3.11), we deduce the relation

$$
\begin{aligned}
& t^{2 \alpha-4 n(M)-4 w_{L}(M)-4 w_{R}(M)}\left\{t^{-2} F_{+}-t^{2} F_{-}\right\} \\
& \quad=\left(t-t^{-1}\right) t^{2 \alpha-4 n(M)-4 w_{L}(M)-4 w_{R}(M)}\left\{F_{0}+t^{8 \alpha+8 w_{R}(M)} F_{\infty}\right\} .
\end{aligned}
$$

Now recall that in (3.1), $\lambda$ is the linking number of $C_{R}$ with $M \cup C_{L}$, and this is $\alpha+w_{R}(M)$. Therefore if we define

$$
a=i t^{-2}, \quad x=i\left(t-t^{-1}\right),
$$

we deduce from (3.12) that

$$
a F_{+}+a^{-1} F_{-}=x\left(F_{0}+a^{-4 \lambda} F_{\infty}\right)
$$

which is the desired result.

Case 2. Again we use the notation described in $\S 2$ for Case 2 . Now we will choose $f_{1}$ and $f_{2}$ to be zero, and also we choose zero framing for every component in $M$. The following relations now follow from explicit computations:

$$
\begin{aligned}
n\left(L_{+}\right) & =n(M)+w_{1}(M)+w_{2}(M)+\beta, \\
n\left(L_{-}\right) & =n(M)+w_{1}(M)+w_{2}(M)+\beta-1, \\
n\left(L_{0}\right) & =n(M)+w_{1}(M)+w_{2}(M), \\
n\left(L_{\infty}\right) & =n(M)-w_{1}(M)+w_{2}(M) .
\end{aligned}
$$

We can now write down the expressions which (1.10) provides for the links $L_{+}, L_{-}, L_{0}, L_{\infty}$, 
$\left(\mathrm{B}_{1}\right)$

$$
\begin{aligned}
t^{-4 n(M)-4 w_{1}(M)-4 w_{2}(M)-4 \beta} F_{+} \\
=g \sum_{J \subset M}(-1)^{|J|}\left\{t^{-6 n(J)} V_{\widetilde{J}}(t)-t^{-6 n(J)-6 w_{1}(J)} V_{\widetilde{J} \cup \widetilde{C}_{1}}(t)\right. \\
\quad-t^{-6 n(J)-6 w_{2}(J)} V_{\widetilde{J} \cup \widetilde{C}_{2}}(t) \\
\left.+t^{-6 n(J)-6 w_{1}(J)-6 w_{2}(J)-6 \beta} V_{\widetilde{J} \cup \widetilde{C}_{+}}(t)\right\} .
\end{aligned}
$$

$$
\begin{aligned}
t^{-4 n(M)-4 w_{1}(M)-4 w_{2}(M)-4 \beta+4} F_{-} \\
=g \sum_{J \subset M}(-1)^{|J|}\left\{t^{-6 n(J)} V_{\widetilde{J}}(t)-t^{-6 n(J)-6 w_{1}(J)} V_{\widetilde{J} \cup \widetilde{C}_{1}}(t)\right. \\
\quad-t^{-6 n(J)-6 w_{2}(J)} V_{\widetilde{J} \cup \widetilde{C}_{2}}(t) \\
\left.+t^{-6 n(J)-6 w_{1}(J)-6 w_{2}(J)-6 \beta+6} V_{\widetilde{J} \cup \widetilde{C}_{-}}(t)\right\} .
\end{aligned}
$$

$$
t^{2 \beta-1-4 n(M)-4 w_{1}(M)-4 w_{2}(M)} F_{0}
$$

$\left(\mathrm{B}_{3}\right)$

$$
\begin{gathered}
=g \sum_{J \subset M}(-1)^{|J|}\left\{t^{6 \beta-3-6 n(J)} V_{\widetilde{J}}(t)\right. \\
\left.-t^{-6 n(J)-6 w_{1}(J)-6 w_{2}(J)} V_{\widetilde{J} \cup \widetilde{C}_{0}}(t)\right\} . \\
t^{-2 \beta+1-4 n(M)+4 w_{1}(M)-4 w_{2}(M)} F_{\infty} \\
=g \sum_{J \subset M}(-1)^{|J|}\left\{t^{-6 \beta+3-6 n(J)} V_{\widetilde{J}}(t)\right. \\
-t^{-6 n(J)+6 w_{1}(J)-6 w_{2}(J)} V_{\left.\widetilde{J} \cup \widetilde{C}_{\infty}(t)\right\} .}
\end{gathered}
$$

Taking linear combinations, we deduce

$$
\begin{aligned}
B_{1}-B_{2}=g \sum_{J \subset M}(-1)^{|J|} t^{-6 n(J)-6 w_{1}(J)-6 w_{2}(J)-6 \beta+3} \\
\times\left\{t^{-3} V_{\widetilde{J} \cup \widetilde{C}_{+}}(t)-t^{3} V \widetilde{J \cup \widetilde{C}_{-}}(t)\right\} .
\end{aligned}
$$

Again using the result of Proposition 2.2, we get

$$
\begin{aligned}
B_{1}-B_{2}=\left(t-t^{-1}\right) g \sum_{J \subset M} & (-1)^{|J|} t^{-6 \beta+3-6 n(J)-6 w_{1}(J)-6 w_{2}(J)} \\
& \times\left\{t^{12 \beta-6+12 w_{1}(J)} V_{\widetilde{J} \cup \widetilde{C}_{\infty}}(t)-V_{\widetilde{J} \cup \widetilde{C}_{0}}(t)\right\} .
\end{aligned}
$$

Furthermore, we also have the relation

$$
\begin{aligned}
t^{-6 \beta+3} B_{3}-t^{6 \beta-3} B_{4} & \\
=g & \sum_{J \subset M}(-1)^{|J|}\left\{t^{6 \beta-3-6 n(J)+6 w_{1}(J)-6 w_{2}(J)} V_{\widetilde{J} \cup \widetilde{C}_{\infty}}(t)\right. \\
& \left.-t^{-6 \beta+3-6 n(J)-6 w_{1}(J)-6 w_{2}(J)} V_{\widetilde{J} \cup \widetilde{C}_{0}}(t)\right\} .
\end{aligned}
$$

Combining (3.16) and (3.17) we deduce

$$
B_{1}-B_{2}=\left(t-t^{-1}\right)\left(t^{-6 \beta+3} B_{3}-t^{6 \beta-3} B_{4}\right) .
$$


Next we substitute the left-hand sides of the expressions into (3.18). After some cancellation this reduces to

$$
t^{-2} F_{+}-t^{2} F_{-}=\left(t-t^{-1}\right)\left\{F_{0}-t^{8 \beta+8 w_{1}(M)-4} F_{\infty}\right\} .
$$

Comparing this with (3.2), we see that $\mu$ is the linking number of $C_{1}$ with $M \cup C_{2}$, and this is $\beta+w_{1}(M)$. Therefore defining $a$ and $x$ by (3.13), we get

$$
a F_{+}+a^{-1} F_{-}=x\left(F_{0}+a^{-4 \mu+2} F_{\infty}\right) .
$$

Therefore we have verified the crossing relations for $F_{L}$ in the expression (1.10). From the first example given in the introduction, we know that $F_{U}$ is one, where $U$ is the unknot. Therefore $F_{L}$ is the Kauffman polynomial.

Finally we prove Theorem 3 . This follows by substituting the right-hand side of (1.10) into (1.18). This gives

$$
\begin{aligned}
\left(t^{1 / 2}\right. & \left.+t^{-1 / 2}\right) \sum_{J \subset L}(-1)^{|J|} t^{3 f\left(J^{c}\right)} \sum_{M \subset J}(-1)^{|M|} t^{3 f(J \backslash M)-6 n(M)} V_{\widetilde{M}}(t) \\
& =\left(t^{1 / 2}+t^{-1 / 2}\right) \sum_{J \subset L}(-1)^{|J|} \sum_{M \subset J}(-1)^{|M|} t^{3 f(L \backslash M)-6 n(M)} V_{\widetilde{M}}(t) \\
& =\left(t^{1 / 2}+t^{-1 / 2}\right) \sum_{M \subset L}(-1)^{|M|} t^{3 f\left(M^{c}\right)-6 n(M)} V_{\widetilde{M}}(t) \sum_{N \subset L \backslash M}(-1)^{|N|+|M|} .
\end{aligned}
$$

However we have the identity

$$
\sum_{N \subset L \backslash M}(-1)^{|N|}=0
$$

unless $L=M$, in which case (3.21) is one. Substituting into (3.20) yields the left-hand side of (1.18), and this proves Theorem 3.

\section{ACKNOWLEDGMENTS}

The author would like to thank J. Fröhlich for many helpful discussions and much encouragement.

\section{REFERENCES}

1. V. F. R. Jones, A polynomial invariant for knots via von Neumann algebras, Bull. Amer. Math. Soc. 12 (1985), 103-110.

2. L. H. Kauffman, New invariants in the theory of knots, Amer. Math. Monthly 95 (1988), 195-242; On knots, Ann. of Math. Stud., no. 115, Princeton Univ. Press, Princeton, N.J., 1987; An invariant of regular isotopy, Trans. Amer. Math. Soc. 318 (1990), 417-471.

3. W. B. R. Lickorish, A relationship between link polynomials, Math. Proc. Cambridge Philos. Soc. 100 (1986), 109-112; L. H. Kauffman, On knots (see [2]).

4. W. B. R. Lickorish and K. C. Millett, The new polynomial invariants of knots and links, Math. Mag. 61 (1988), 3-23.

5. V. F. R. Jones, Hecke algebra representations of braid groups and link polynomials, Ann. of Math. 126 (1987), 335-388.

6. T. Kohno, Monodromy representations of braid groups and Yang-Baxter equations, Ann. Inst. Fourier (Grenoble) 37 (1987), 139-160.

7. V. G. Turaev, The Yang-Baxter equation and invariants of links, LOMI preprint E-3-87, Steklov Math. Inst. Leningrad, 1987.

8. J . Fröhlich and C. King, The Chern-Simons theory and knot polynomials, Comm. Math. Phys. 126 (1989), 167-200. 
9. E. Witten, Quantum field theory and the Jones polynomial, Comm. Math. Phys. 121 (1989), 351-400.

10. V. G. Knizhnik and A. B. Zamolodchikov, Current algebra and Wess-Zumino models in two dimensions, Nuclear Phys. B247 (1984), 83-103.

11. J. Fröhlich and C. King, Two-dimensional conformal field theory and three-dimensional topology, Internat. J. Modern Phys. A 4 (1989), 5321-5400.

12. J. Birman and T. Kanenobu, Jones' braid-plat formula and a new surgery triple, Proc. Amer. Math. Soc. 102 (1988), 687-695.

Department of Mathematics, Cornell University, Ithaca, New York 14853

Current address: Department of Mathematics, Northeastern University, Boston, Massachusetts 02115 